\title{
Tecnologias para a autoeficácia materna na prevenção da diarreia infantil: ensaio clínico
}

\author{
Technologies for maternal self-efficacy in preventing childhood diarrhea: a clinical trial \\ Tecnologías para la autoeficacia materna en la prevención de la diarrea infantil: ensayo clínico
}

\author{
Elizamar Regina da Rocha Mendes ${ }^{1}$ io https://orcid.org/0000-0002-1552-5622 \\ Leidiane Minervina Moraes de Sabino² id https://orcid.org/0000-0003-2938-870X \\ Paulo César de Almeida ${ }^{1}$ ID https://orcid.org/0000-0002-2867-802X \\ Emanuella Silva Joventino Melo² io https://orcid.org/0000-0001-9786-5059 \\ Jardeliny Corrêa da Penha ${ }^{3}$ io https://orcid.org/0000-0001-5956-9072 \\ Silvana Santiago da Rocha ${ }^{3}$ io https://orcid.org/0000-0002-1325-9631 \\ Lorena Pinheiro Barbosa ${ }^{1}$ io https://orcid.org/0000-0002-8006-7517
}

Mendes ER, Sabino LM, Almeida citar: Penha JC, Rocha SS, et al. Tecnologias para a utoeficácia materna na prevenção da diarreia infantil: ensaio clínico. Acta Paul Enferm. 2021;34:EAPE03232.

DOI

http://dx.doi.org/10.37689/actaape/2021A003232

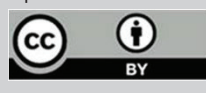

Diarreia infantil; motivacional; Educação em saúde; Enfermagem

Keywords

Children's diarrhea; Self-efficacy; Motivationa interview; Health education; Nursing

\section{Descriptores}

Diarrea infantil: Autoeficacia; Entrevista motivacional; Educación para la salud; Enfermería

\section{Submetido \\ 6 de Dezembro de 2019 \\ Aceito \\ 17 de Junho de 2020}

Autor correspondente

Elizamar Regina da Rocha Mendes E-mail: elizamarregina@hotmail.com

\section{Resumo}

Objetivo: Avaliar os efeitos de duas tecnologias educativas, vídeo e cartilha, associadas à entrevista motivacional para promover a autoeficácia materna na prevenção da diarreia infantil.

Métodos: Ensaio clínico com 122 mães de crianças menores de 5 anos, randomizadas aleatoriamente em dois grupos: vídeo educativo ("Diarreia Infantil: você é capaz de prevenir") aliado à Entrevista Motivacional Breve ou cartilha ("Você é capaz de prevenir a diarreia no seu filho!") aliada à Entrevista Motivacional Breve. A coleta ocorreu presencialmente no primeiro contato com as mães, na unidade de atenção primária à saúde, aplicando-se a Escala de Autoeficácia Materna para prevenção da Diarreia Infantil e um formulário sociodemográfico. 0 segundo e terceiro momentos de coleta de dados ocorreram por telefone, após um e dois meses, respectivamente, utilizando-se a escala e um formulário de investigação da diarreia.

Resultados: Constatou-se que um mês após as intervenções, a maioria das participantes dos dois grupos foram consideradas com autoeficácia elevada para prevenção da diarreia infantil, sendo 78,4\% das mães no grupo vídeo e entrevista motivacional e; 83,0\% no grupo cartilha e entrevista motivacional. Dois meses após as intervenções, as médias dos escores de autoeficácia materna elevaram-se e tiveram redução nos casos de diarreia infantil, em ambos os grupos.

Conclusão: Comprovou-se que essas tecnologias educativas aliadas à entrevista motivacional breve elevaram a autoeficácia para prevenir a diarreia infantil. Logo, podem ser utilizadas pelos enfermeiros que atuam na Atenção Primária à Saúde, como uma ferramenta educativa.

Registro Brasileiro de Ensaios Clínicos: RBR-6974xz

\section{Abstract}

Objective: To assess the effects of two educational technologies, video and booklet, associated with Motivational Interviewing to promote maternal self-efficacy in preventing childhood diarrhea.

Methods: A clinical trial conducted with 122 mothers of children under 5 years old; mothers were randomly randomized into two groups: educational video ("Child's diarrhea: you are able to prevent it") combined with Brief Motivational Interviewing or booklet ("You are able to prevent diarrhea in your child!") combined with Brief Motivational Interviewing. Data collection took place in the first contact with the mothers at the Primary Health Care unit; the Maternal Self-Efficacy Scale for preventing childhood diarrhea and a sociodemographic form were applied. The second and third moments of data collection occurred through telephone, after one and two months, respectively, using the scale and a form for investigating diarrhea. 
Results: It was found that one month after the interventions, most of the participants in both groups were considered to have high self-efficacy in preventing childhood diarrhea, with $78.4 \%$ of mothers in the video and Motivational Interviewing group; $83.0 \%$ in the booklet and Motivational Interviewing group. Two months after the interventions, mean maternal self-efficacy scores increased and decreased in cases of childhood diarrhea in both groups.

Conclusion: It has been proved that these educational technologies, combined with Brief Motivational Interviewing, increased self-efficacy to prevent childhood diarrhea. Therefore, they can be used by nurses working at Primary Health Care as an educational tool.

\section{Resumen}

Objetivo: Evaluar los efectos de dos tecnologías educativas, video y cartilla, junto con la encuesta motivacional, para promover la autoeficacia materna en la prevención de la diarrea infantil.

Métodos: Ensayo clínico con 122 madres de niños menores de 5 años, aleatorizadas en dos grupos: video educativo ("Diarrea infantil: tú puedes prevenirla") junto con la encuesta motivacional breve o cartilla (“iTú puedes prevenir la diarrea de tu hijo!") junto con la encuesta motivacional breve. La recolección se realizó presencialmente en el primer contacto con las madres, en la unidad de atención primaria de salud, mediante la implementación de la Escala de Autoeficacia Materna para la Prevención de la Diarrea Infantil y un formulario sociodemográfico. El segundo y tercer momento de recolección de datos fue por teléfono, luego de uno y dos meses, respectivamente, mediante la escala y un formulario de investigación de la diarrea.

Resultados: Se constató que, un mes después de las intervenciones, la mayoría de las participantes de los dos grupos fueron consideradas con autoeficacia elevada para la prevención de la diarrea infantil, de las cuales el 78,4 \% pertenecían al grupo video y encuesta motivacional y el 83,0 \% al grupo cartilla y encuesta motivacional. Dos meses después de las intervenciones, el promedio de la puntuación de autoeficacia materna aumentó y se redujeron los casos de diarrea infantil en ambos grupos.

Conclusión: Se comprobó que estas tecnologías educativas, junto con la encuesta motivacional breve, aumentaron la autoeficacia para prevenir la diarrea infantil. Por lo tanto, pueden ser utilizadas por los enfermeros que actúan en la atención primaria de salud, como herramienta educativa.

\section{Introdução}

A diarreia infantil, mesmo sendo uma doença evitável e tratável, continua sendo uma das principais causas de consultas, hospitalizaçóes, atendimentos em emergências e óbitos em menores de cinco anos de idade. Estima-se que ocorram, anualmente, 2,5 bilhóes de casos em crianças desta faixa etária, levando a 57 óbitos por 100.000 habitantes e representando a segunda maior causa de morbimortalidade mundial em crianças nos países em desenvolvimento. ${ }^{(1-4)}$

A doença diarreica aguda está associada a múltiplos fatores relacionados às condiçôes socioeconômicas, sanitárias, culturais e comportamentais. ${ }^{(5,6)}$ Nos países de baixa renda, crianças de até três anos apresentam, em média, três episódios diarreicos a cada ano. Cada episódio priva a criança da nutrição necessária para o crescimento, além de levar à desidratação, tornando-se um ciclo, já que as crianças desnutridas são mais propensas a adoecer por diarreia. ${ }^{(7)}$

Estudos evidenciam uma redução na incidência da diarreia, por meio das seguintes medidas: incentivo ao aleitamento materno, ${ }^{(8)}$ vacina rotavírus, ${ }^{(9,10)}$ saneamento básico, ${ }^{(11)}$ comportamento materno. ${ }^{(12)}$ Além destas, intervençóes educativas podem contribuir para o aumento da confiança materna em prevenir a diarreia infantil. ${ }^{(12)}$
Sabe-se que o conhecimento materno e atitudes adequadas no manejo e tratamento da diarreia são essenciais para prevenir o agravo. No entanto, apenas conhecer os fatores que causam a diarreia não significa uma adesão a comportamentos desejáveis, pois, mesmo as mães com conhecimento insuficiente acerca dos fatores de risco, mas que acreditam que podem manter seus filhos saudáveis são mais propensas a realizarem comportamentos preventivos contra a doença. ${ }^{(13,14)}$ Logo, não basta o indivíduo saber que determinado padrão de comportamento pode ajudá-lo a atingir um objetivo específico, é preciso que ele também se sinta capaz de executá-lo. ${ }^{(15)}$

Diante disso, a atuação do enfermeiro é indispensável, visto que ele deve implementar medidas educativas, direcionadas às mães, em prol da promoção da saúde, da autoeficácia e da prevenção da diarreia infantil. Nesse ínterim, autoeficácia é um conceito incorporado à Teoria Social Cognitiva (TSC) sendo definida como julgamentos ou crenças que as próprias pessoas fazem sobre suas capacidades para executar ações necessárias para obter um resultado desejado. ${ }^{(16)}$ Ademais, reconhece-se que níveis elevados de autoeficácia identificados nas mães por meio da Escala de Autoeficácia Materna para Prevenção da Diarreia Infantil (EAPDI) mostram-se um fator protetor para a diarreia infantil. ${ }^{(17,18)}$

Nesse sentido, foram desenvolvidas tecnologias educativas baseadas na TSC com o objetivo de ele- 
var a autoeficácia materna para prevenção da diarreia infantil. ${ }^{(12,18)}$ Sabe-se que a prática de cuidar do enfermeiro requer o uso de tecnologias, com ênfase nas relacionais, uma vez que são essenciais para um maior diálogo e para fortalecer uma relação horizontal entre profissional e paciente. ${ }^{(19)}$ A exemplo disso, cita-se a Entrevista Motivacional que se trata de um método de aconselhamento breve, centrado no indivíduo com o objetivo de alcançar suas motivaçôes para mudanças de comportamento. ${ }^{(20)}$

Além disso, revisão sistemática constatou que intervençóes com tecnologias associadas à Entrevista Motivacional possuem eficácia maior, em comparação com aquelas realizadas isoladamente. ${ }^{(20)}$ Assim, acredita-se que o uso de tecnologias educativas leves, como a Entrevista Motivacional Breve, que permitem uma relação dialógica entre profissional e paciente, podem promover mudanças de comportamento, sobretudo se aliadas a outras tecnologias, tais como vídeos e cartilhas educativas, sendo capazes de elevar os níveis de autoeficácia materna em prevenir a diarreia infantil, além de auxiliar os enfermeiros nas orientaçóes às mães sobre a prevenção da diarreia, tornando-as mais seguras e capazes de cuidar dos filhos e, consequentemente, reduzindo a morbimortalidade por esse agravo.

Nesse sentido, o estudo objetivou comparar a eficácia da Entrevista Motivacional Breve (EMB) combinada ao vídeo educativo "Diarreia Infantil: Você é capaz de prevenir" e da EMB aliada à cartilha educativa "Você é capaz de prevenir a diarreia no seu filho!” sobre autoeficácia materna para prevenir a diarreia infantil.

\section{Métodos}

Trata-se de um estudo experimental, do tipo ensaio clínico controlado randomizado em cluster, registrado e aprovado no Registro Brasileiro de Ensaio Clínico (Rebec), com número de registro RBR6974xz, com dois grupos paralelos, duplo cego, desenvolvido com mães de crianças menores de cinco anos cadastradas em Unidades de Atenção Primária à Saúde (UAPS), em Fortaleza, Ceará, Brasil, entre julho e setembro de 2015.
Foram consideradas elegíveis mães que tivessem pelo menos um filho menor de cinco anos de idade cadastrado em uma das UAPS selecionadas e que possuísse contato telefônico. Foram excluídas mães de criança diagnosticada com intolerância à lactose ou com alguma outra patologia cuja sintomatologia fosse diarreia crônica, bem como mãe que apresentasse alguma limitação cognitiva aparente ou que não soubesse ler. Como critérios de descontinuidade: desistência da mãe, mudança de número de telefone e falecimento da mãe ou da criança.

O tamanho da amostra foi baseado na premissa de que, para o teste de qui-quadrado, o número mínimo em cada célula deveria ser de aproximadamente 10 observaçóes. Haja vista que a maior tabela com a escala de autoeficácia versus grupo foi de cinco categorias e dois grupos, portanto, 10 células. (21) Assim, foram necessários $10 \times 10=100$ indivíduos, com acréscimo de 22\%, ficaram 122 mães, de modo que cada grupo foi composto por 61 mães.

Fixou-se um erro do tipo I em 5\%, supondo que, a probabilidade de se rejeitar que os efeitos das tecnologias educativas associadas serem diferentes, quando elas poderiam ser iguais, ou seja, nesse caso, seria no máximo de 5\%. Fixou-se um erro do tipo II em $20 \%$, supondo que, a probabilidade de se aceitar que os efeitos das tecnologias educativas associadas, quando elas poderiam ser diferentes, seria no máximo em $20 \%$.

A randomização ocorreu por conglomerados ou cluster. A alocação randômica dos conglomerados foi realizada considerando-se os bairros de Fortaleza, com posterior alocação aleatória simples por meio de uma tabela de números aleatórios para escolha das UAPS, bem como para a seleção das mães que compuseram cada grupo. Assim, cada mãe de cada conglomerado pôde ter iguais chances de fazer parte da amostra do estudo. Desta maneira, um conglomerado fez parte do grupo intervenção com vídeo e EMB e o outro, do grupo intervenção com cartilha e EMB, evitando-se que mães de um mesmo bairro compusessem os dois grupos, a fim de reduzir ou eliminar compartilhamento de informaçôes sobre as intervençóes nos grupos. ${ }^{(22)}$

O estudo foi duplo cego tendo em vista que os pesquisadores responsáveis pelo acompanhamento por te- 
lefone não tinham conhecimento para qual dos grupos estava ligando, bem como procedeu-se o cegamento para o estatístico que realizou a análise dos dados.

A coleta de dados ocorreu em três fases: I - primeira avaliação com a EAPDI e aplicação do formulário sociodemográfico (nas UAPS); fase II - intervençôes educativas e segunda avaliação da EAPDI (nas UAPS) e; fase III - acompanhamento por telefone por 2 meses.

A Fase I ocorreu nas UAPS, onde as mães foram convidadas a participarem do estudo enquanto aguardavam por consulta de puericultura, vacinação ou outro atendimento. Após o aceite, foram aplicados, em ambos os grupos, a EAPDI e o formulário, contendo questôes sociodemográficas e de investigação da ocorrência de diarreia infantil. O tempo de duração estimado desse momento foi aproximadamente, 20 minutos, sendo 6 minutos para a EADPI e 14 minutos para o formulário sociodemográfico.

A EAPDI é um instrumento brasileiro que serve para avaliar a confiança das máes e/ou cuidadores sobre a realização de cuidados para prevenir a diarreia infantil. A escala é composta por 24 itens, divididos em dois domínios, práticas gerais/alimentares e higiene da família. Os escores da escala classificam-se em: baixa autoeficácia (escores $\leq 109$ ), moderada autoeficácia (escores entre 110 e 114 pontos) e elevada autoeficácia (escores $\geq 115$ ). O Índice de Validade de Conteúdo (IVC) deste instrumento é de 0,96 e $\alpha$ de Cronbach de 0,84. (23) $^{23}$

Quanto ao formulário sociodemográfico e sanitário, foram investigadas as seguintes variáveis: idade materna, escolaridade, estado civil, número de filhos, número de pessoas na residência e condições sanitárias e de moradia. E no que concerne à investigação da diarreia, as mães foram questionadas sobre a ocorrência recente de diarreia na criança e o manejo materno da doença.

$\mathrm{Na}$ fase II, que ocorreu no mesmo dia da fase I, procederam-se as intervençóes educativas nas UAPS: as mães do grupo vídeo e EMB assistiram individualmente, uma única vez, ao vídeo educativo intitulado "Diarreia Infantil: você é capaz de prevenir", por meio de notebook e fones de ouvido. Já as máes do grupo cartilha e EMB foram orientadas a lerem individualmente a cartilha educativa.
Ambas as tecnologias (vídeo e cartilha) abordam a promoção da autoeficácia materna para prevenção da diarreia infantil, tendo sido elaboradas a partir do referencial teórico da Autoeficácia de Bandura ${ }^{(16)}$ e dos itens da EAPDI, onde são expostos textos e imagens no ambiente doméstico e na unidade de atenção primária à saúde, abordando cuidados para prevenção e manejo da diarreia infantil. ${ }^{(23)}$ Além disso, as tecnologias foram validadas no Brasil por 23 e 30 juízes de conteúdo, respectivamente, e com a população-alvo. ${ }^{(12,17)} \mathrm{O}$ vídeo apresentou IVC geral de $0,97,{ }^{(17)}$ e a cartilha com IVC global de 0,88 . (12) Sendo verificado que os materiais estavam adequados a serem utilizados na prática assistencial.

Após a aplicação das referidas tecnologias educativas, cada mãe participou da EMB. Esta tem a característica de incentivar a autonomia e capacidade de escolha do indivíduo, podendo ser uma intervenção a ser utilizada de forma isolada ou em associação com outras tecnologias.

A EMB desta pesquisa foi baseada nas orientaçôes apresentadas no vídeo ou na cartilha educativos, a depender do grupo e direcionada para as necessidades de cada participante, enfocando sobretudo os itens que as mães atingiram menor pontuação na aplicação prévia da EAPDI, ou seja, nos itens que elas se sentiram menos confiantes para executar em seu cotidiano. Desse modo, o intuito da EMB era elevar a autoeficácia das participantes.

Destaca-se ainda que a EMB foi previamente semiestruturada, para que pudesse ter a mesma conduta com todas as participantes, em que se buscou abordar as quatro crenças de autoeficácia (experiências de êxito, experiências vicárias, persuasão verbal e estados fisiológicos) e foi realizada conforme esse roteiro: 1 . apresentação, aproximação e permissão; 2 . informação; 3. evocação inicial; e 4. evocação final.

Imediatamente após a aplicação das tecnologias e EMB, ainda na UAPS, foi realizada a segunda avaliação da autoeficácia por meio da EAPDI.

A fase III iniciou quinze dias após as intervençóes e consistiu em um acompanhamento quinzenal por contato telefônico nos dois grupos ao longo de dois meses após a fase I e II. Portanto, para a coleta de dados foram utilizados dois instrumentos: a EAPDI e o formulário reduzido de investigação da diarreia infantil. 
Os resultados seguem apresentados em tabelas, contendo medidas de tendência central, odds ratio, testes de qui-quadrado e razão de verossimilhança. Para a comparação das médias, foram utilizados os testes t de Student e Mann-Whitney. Adotou-se como estatisticamente significante o $\mathrm{p}<0,05$. Os dados foram processados no SPSS 20, licença número 10101131007.

O estudo foi aprovado pelo Comitê de Ética em Pesquisa da Universidade Federal do Ceará - UFC, com o Parecer 1.116.855, inscrito e aprovado no Registro Brasileiro de Ensaio Clínico (Rebec), com número de registro RBR-6974xz. Respeitaram-se os princípios éticos para pesquisas envolvendo seres humanos de acordo com a Resolução 466/12 do Conselho Nacional de Saúde. A coleta de dados teve início mediante assinatura de um termo de consentimento livre e esclarecido (TCLE), expedido em duas vias, ficando uma com as participantes do estudo.

\section{Resultados}

A amostra total do estudo foi composta por 122 mães de crianças em idade inferior a cinco anos de idade, distribuídas em dois grupos, de modo que 61 compuseram o grupo intervenção com vídeo educativo e entrevista motivacional breve, e 61, o grupo intervenção com cartilha educativa e entrevista motivacional breve. Ao longo da pesquisa, devido aos critérios de descontinuidade, houve algumas perdas em ambos os grupos, conforme ilustrado na figura 1 .

$\mathrm{Na}$ tabela 1 é possível observar que as variáveis sociodemográficas e sanitárias, não apresentaram diferença estatística significativa na linha de base, portanto, os dois grupos eram homogêneos no que diz respeito a características demográficas, socioeconômicas e sanitárias: escolaridade $(\mathrm{p}=0,196)$, estado civil ( $p=0,185)$, número de filhos vivos $(p=0,219)$, número de pessoas na residência $(\mathrm{p}=0,189)$, tipo de casa $(\mathrm{p}=1,000)$, tipo de piso $(\mathrm{p}=0,131)$, destino do lixo $(\mathrm{p}=0,154)$, tipo de sanitário $(\mathrm{p}=0,764)$ e moscas no domicílio ( $\mathrm{p}=0,904)$.

A maioria das mães do grupo vídeo e EMB e; do grupo cartilha e EMB tinha, respectivamente, o ensino médio concluído $(54,1 \% ; 65,6 \%)$, era casada $(78,7 \% ; 75,4 \%)$, tinha dois ou mais filhos $(63,9 \%$; $54,2 \%)$, mais de 4 pessoas morando na mesma residência $(80,0 \% ; 68,4 \%)$, morava em casas com reboco $(93,4 \% ; 93,4 \%)$, com piso de cerâmica $(70,5 \% ; 57,4 \%)$ e o destino do lixo proveniente da

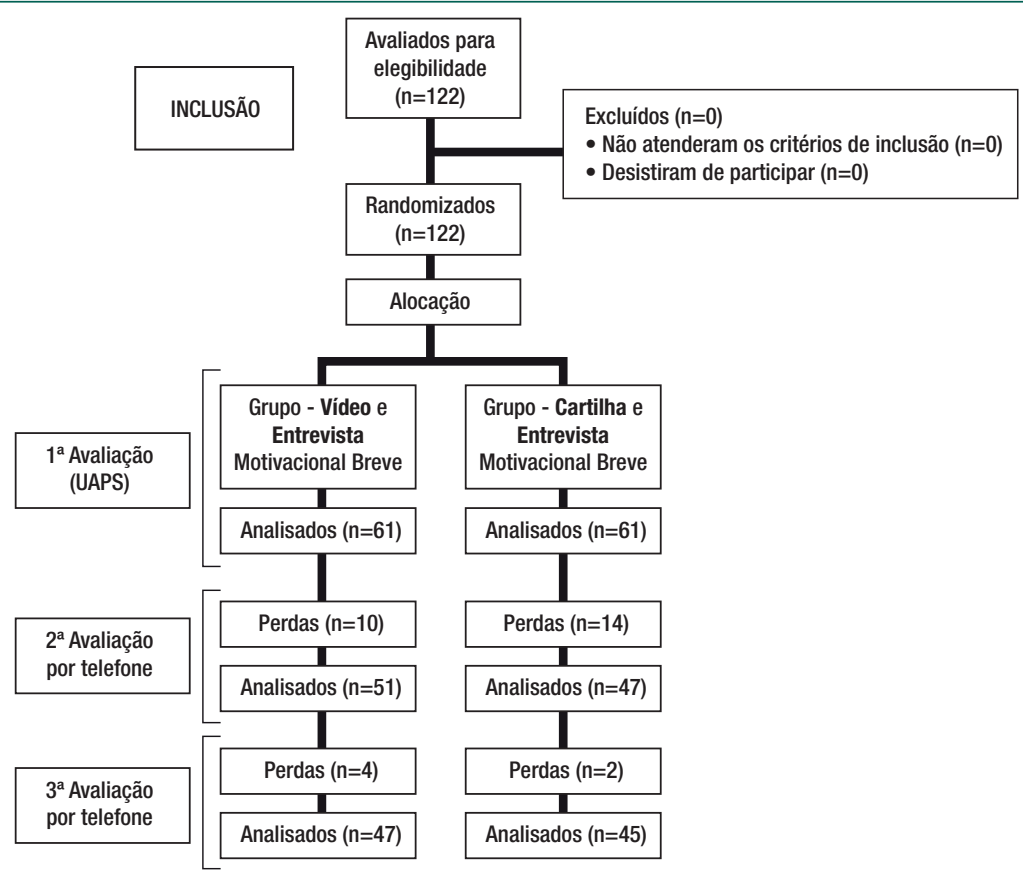

Figura 1. Diagrama representativo do fluxo das mães em cada fase do estudo 
Tabela 1. Características sociodemográficas das mães e sanitárias dos domicílios segundo os grupos da pesquisa

\begin{tabular}{|c|c|c|c|}
\hline \multirow[b]{2}{*}{$\begin{array}{l}\text { Variáveis sociodemográficas e } \\
\text { sanitárias }\end{array}$} & \multicolumn{3}{|c|}{ Grupos } \\
\hline & $\begin{array}{l}\text { Vídeo e EMB }{ }^{\star \star} \\
\mathrm{n}(\%)\end{array}$ & $\begin{array}{c}\text { Cartilha e EMB }{ }^{\star \star} \\
\mathrm{n}(\%)\end{array}$ & $p$-value ${ }^{*}$ \\
\hline Escolaridade da mãe & & & 0,196 \\
\hline Fundamental & $28(45,9)$ & $21(34,4)$ & \\
\hline Ensino Médio & $33(54,1)$ & $40(65,6)$ & \\
\hline Estado Civil & & & 0,185 \\
\hline União consensual/casada & $48(78,7)$ & $46(75,4)$ & \\
\hline Solteira & $13(21,3)$ & $15(24,6)$ & \\
\hline Número de Filhos & & & 0,219 \\
\hline 1 & $22(36,1)$ & $27(45,8)$ & \\
\hline 2 & $18(29,5)$ & $20(33,9)$ & \\
\hline 3 ou mais & $21(34,4)$ & $12(20,3)$ & \\
\hline Número de pessoas na residência & & & 0,189 \\
\hline 2 a 3 & $11(19,0)$ & $19(31,7)$ & \\
\hline 4 a 5 & $33(56,9)$ & $25(41,7)$ & \\
\hline 6 ou mais & $14(24,1)$ & $16(26,7)$ & \\
\hline Tipo de casa & & & 1,000 \\
\hline Com reboco & $57(93,4)$ & $57(93,4)$ & \\
\hline Sem reboco & $4(6,6)$ & $4(6,6)$ & \\
\hline Tipo de piso & & & 0,131 \\
\hline Cerâmica & $43(70,5)$ & $35(57,4)$ & \\
\hline Cimento & $18(29,5)$ & $26(42,6)$ & \\
\hline Destino lixo & & & 0,154 \\
\hline Coleta pública & $61(100)$ & $59(96,7)$ & \\
\hline Outros & $0(0)$ & $0(0)$ & \\
\hline Tipo de Sanitário & & & 0,764 \\
\hline Com descarga & $49(84,5)$ & $51(86,4)$ & \\
\hline Sem descarga & $9(15,5)$ & $8(13,6)$ & \\
\hline Moscas no domicílio & & & 0,904 \\
\hline $\operatorname{Sim}$ & $46(78)$ & $47(77)$ & \\
\hline Não & $13(22)$ & $14(23)$ & \\
\hline
\end{tabular}

*Teste de Qui-quadrado de Pearson; **EMB - Entrevista Motivacional Breve

coleta pública $(100 \% ; 96,7 \%)$. A tabela 2 ilustra a média da escala total e dos dois domínios da EAPDI comparando-se os dois grupos do estudo. Os domínios da EAPDI referem-se a comportamentos gerais e alimentares, bem como de higiene a serem adotados com a criança e família.

Antes das intervençóes, observa-se proximidade nas médias da escala total, com apenas 2,0 pontos de diferença entre os dois grupos. Essa diferença mínima na linha de base tornou possível afirmar que os grupos são semelhantes. Ademais, em nenhum dos momentos investigados existiu associação estatisticamente significativa entre os mesmos, ratificando essa semelhança entre os grupos (Tabela 2).

Um mês após as intervençôes, houve aumento da média dos escores da EAPDI, nos dois grupos, no grupo do vídeo e EMB, bem como no grupo cartilha e EMB, tendo, respectivamente, um aumento de 7 pontos, e 4,9 pontos. Constata-se, portanto,
Tabela 2. Comparativo entre os grupos das médias da escala total e dos domínios antes e após a intervenção

\begin{tabular}{lccccc}
\hline \multirow{2}{*}{ Escores da autoeficácia } & \multicolumn{5}{c}{ Grupos } \\
& $\overline{\mathbf{X}}$ & $\pm \mathrm{DP}$ & $\overline{\mathrm{X}}$ & $\pm \mathrm{DP}$ & \\
\hline Antes da intervenção & & & & & \\
Escala total & 110,0 & $\pm 8,7$ & 112,0 & $\pm 6,6$ & 0,131 \\
Práticas gerais/alimentares & 40,6 & $\pm 3,7$ & 41,2 & $\pm 3,0$ & 0,357 \\
Higiene da família & 69,4 & $\pm 5,0$ & 66,4 & $\pm 3,9$ & 0,061 \\
1 mês depois da intervenção & & & & & \\
Escala total & 116,9 & $\pm 4,4$ & 116,9 & $\pm 5,4$ & 0,951 \\
Práticas gerais/alimentares & 43,8 & $\pm 1,9$ & 43,7 & $\pm 2,1$ & 0,731 \\
Higiene da família & 73,1 & $\pm 2,9$ & 73,2 & $\pm 3,8$ & 0,906 \\
2 meses depois da intervenção & & & & & \\
Escala total & 117,4 & $\pm 4,1$ & 115,9 & $\pm 6,9$ & 0,253 \\
Práticas gerais/alimentares & 43,7 & $\pm 2,2$ & 43,4 & $\pm 2,5$ & 0,670 \\
Higiene da família & 73,7 & $\pm 2,4$ & 72,5 & $\pm 4,5$ & 0,132 \\
\hline *Teste qui-quadrado; **Entrevista Motivacional Breve & & & &
\end{tabular}

${ }^{*}$ Teste qui-quadrado; **Entrevista Motivacional Breve

que houve um aumento significativo nos escores da EAPDI no grupo vídeo e EMB em relação ao grupo cartilha e EMB. Após 2 meses, o grupo vídeo e EMB, permaneceu praticamente com a mesma pontuaçáo, passando de 117,0 para 117,4 pontos, enquanto, que o grupo da cartilha e EMB, diminuiu de 116,9 para 116,0 pontos.

A tabela 3 apresenta o efeito das intervençóes educativas na ocorrência da diarreia infantil. Observa-se que não existiu associação estaticamente significante entre a ocorrência da diarreia e os grupos.

Tabela 3. Comparação do efeito das intervenções educativas na ocorrência de episódios diarreicos na criança

\begin{tabular}{|c|c|c|c|c|c|}
\hline \multirow{3}{*}{ Episódio diarreico } & \multicolumn{4}{|c|}{ GRUPOS } & \multirow{3}{*}{$p$-value ${ }^{* x}$} \\
\hline & \multicolumn{2}{|c|}{ Vídeo e EMB } & \multicolumn{2}{|c|}{ Cartilha e EMB } & \\
\hline & $\mathrm{n}(\%)$ & $\begin{array}{c}\mathrm{RC}^{*} \\
\text { (IC 95\%) }\end{array}$ & $\mathrm{n}(\%)$ & $\begin{array}{c}\mathrm{RC}^{\star} \\
(\mathrm{IC} 95 \%)\end{array}$ & \\
\hline Antes da intervenção & & & & & 0,835 \\
\hline $\operatorname{Sim}$ & $34(57,6)$ & 1,04 & $34(55,7)$ & 0,96 & \\
\hline Não & $25(42,4)$ & $0,71-1,50$ & $27(44,3)$ & $0,67-1,37$ & \\
\hline $\begin{array}{l}1 \text { mês após a } \\
\text { intervenção }\end{array}$ & & & & & 0,942 \\
\hline $\operatorname{Sim}$ & $1(1,9)$ & 0,95 & $1(2,1)$ & 1,05 & \\
\hline Não & $51(98,1)$ & $0,23-3,85$ & $46(97,9)$ & $0,26-4,28$ & \\
\hline $\begin{array}{l}2 \text { meses após a } \\
\text { intervenção }\end{array}$ & & & & & 0,519 \\
\hline $\operatorname{Sim}$ & $1(2,1)$ & 0,63 & $2(4,5)$ & 1,39 & \\
\hline Não & $46(97,9)$ & $0,12-3,19$ & $42(95,5)$ & $0,60-3,20$ & \\
\hline
\end{tabular}

*Razão de chance/intervalo de confiança; **Teste qui-quadrado

Em linha de base, mais da metade das mães de cada grupo, informou que seu filho já teve diarreia. Antes da intervenção, notou-se que as chances de as crianças terem diarreia foi de 1,04 vezes maior 
no grupo vídeo e EMB, do que no grupo cartilha e EMB. Identificou-se uma diminuiçẫo dos casos de diarreia ao longo dos dois meses nos dois grupos. Um mês após a intervenção, observa-se nos dois grupos uma considerável redução nos episódios diarreicos. No grupo vídeo apenas 1,9\% das crianças tiveram diarreia, e no grupo cartilha, $2,1 \%$. Os baixos percentuais mantiveram-se no segundo mês, apenas $2,1 \%$ das mães relataram a ocorrência da diarreia. De modo semelhante ocorreu com o grupo da cartilha e EMB, que após os dois meses da intervenção, apenas 4,5\% das crianças tiveram diarreia. Esses dados indicam que cada uma das intervençôes educativas associadas à entrevista motivacional breve, foram capazes de produzir resultados significativos no que se refere à diminuição da ocorrência da diarreia infantil.

\section{Discussão}

A prevenção da diarreia infantil está intimamente relacionada com açóes executadas pelos cuidadores das crianças, sobretudo pelas mães, já que com base na influência dos cuidados maternos prestados a esta população, admite-se o seu protagonismo na redução dos índices de diarreia infantil. ${ }^{(24)}$ Assim, é indispensável que as mesmas sejam orientadas quanto à prevenção de diarreia infantil e sintam-se capazes de prestar um cuidado adequado aos seus filhos, pois acredita-se que mães com autoeficácia elevada e instruídas, se sentirão capazes de reconhecerem os fatores de risco, de prevenirem e de tomarem condutas adequadas diante dessa patologia nas crianças, promovendo, dessa forma, um cuidado de maior qualidade aos seus filhos.

Pesquisa realizada no Ceará, Brasil com mães de crianças menores de cinco anos de idade identificou que as condiçóes socioeconômicas, comportamentais e conhecimentos maternos teve associação com a ocorrência de diarreia infantil e com a autoeficácia para prevenção da diarreia infantil. ${ }^{(24)}$

Corroborando com os dados das tabelas 1 e 2, ensaio clínico randomizado, realizado em Fortaleza, Ceará, Brasil, com 133 mães de crianças menores de cinco anos, sendo 70 alocadas no grupo de com- paração e 63 no grupo de intervenção identificou que, após a intervenção com vídeo educativo, houve elevação dos escores de autoeficácia em prevenir a diarreia e redução dos episódios diarreicos. ${ }^{(25)}$

Diante desses achados percebe-se a importância dos profissionais e pesquisadores desenvolverem tecnologias e estratégias educativas que fortaleçam o conhecimento e a autoeficácia dos indivíduos, incentivando as mães e/cuidadores a adotarem comportamentos saudáveis a fim de prevenir a diarreia de seus filhos.

Em consonância com os resultados do presente estudo, pesquisas realizadas com a utilização de materiais educativos, pautados na Teoria da Autoeficácia de Bandura, têm evidenciado aumento da autoeficácia com o público participante, como um ensaio clínico randomizado realizado no estado do Ceará, Brasil com 2 grupos (comparação e intervenção), compostos por 180 máes de crianças menores de 5 anos, constatou-se que o grupo que participou da intervenção com vídeo educacional teve um efeito significativo na autoeficácia materna em prevenir a diarreia. ${ }^{(17)}$

Ensaio clínico randomizado, realizado com 153 gestantes na cidade de Miandoab, Irã, em 2015 a 2016, com três grupos, sendo um grupo controle e dois grupos intervenção, em que um recebeu a intervenção com o uso da cartilha educativa e o outro com um e-learning, ambos sobre a autoeficácia do parto. A análise dos dados evidenciou que os dois grupos intervenção foram eficazes ao apresentaram maior autoeficácia quando comparados ao grupo controle, com achados estatisticamente significantes. ${ }^{(26)}$

Estudo quase experimental realizado em Flandres, na Bélgica, com o objetivo de avaliar o efeito de um vídeo online sobre promoção de atividade física e dieta saudável em crianças e da autoeficácia dos pais para promover essas práticas com seus filhos, a fim de prevenir o sobrepeso ou a obesidade. O grupo intervenção assistiu ao vídeo por 4 semanas e obteve aumento da autoeficácia parental relacionada à promoção da prática de atividade física e dieta saudável dos filhos, principalmente entre os pais de crianças de 6 a 9 anos de idade. ${ }^{(27)}$

Pelos achados do presente ECR e demais investigaçôes, infere-se que quando as mães se sentem ca- 
pazes de cuidar adequadamente dos filhos, há uma promoção da saúde das crianças e redução da morbimortalidade das mesmas, especialmente por diarreia, pois, a crença de um indivíduo em sua própria capacidade é um bom preditor de motivação e de mudança comportamento. ${ }^{(16)}$

Ambas as tecnologias associadas à entrevista motivacional breve, trouxeram resultados para o aumento nos escores da autoeficácia materna em prevenir a diarreia infantil. Embora não tenham sido encontrados estudos anteriores com intervençôes educativas aliadas à Entrevista Motivacional Breve voltados para prevenção da diarreia, outra pesquisa que incorporou a Entrevista Motivacional atestou a eficácia da técnica por meio de resultados que evidenciaram melhorias nas condiçóes de saúde. ${ }^{(28)}$

Nesse sentido acredita-se que materiais educativos como álbum seriado, cartilha, manuais, folhetos informativos e vídeos, são tecnologias duras válidas na transmissão da mensagem entre educador e educando. ${ }^{(29)}$ No entanto, para serem considerados eficazes, precisam apresentar vocabulário sucinto, relevante, bem elaborado, atrativo e de fácil compreensão. ${ }^{(30)}$ Ressalta-se que independente do recurso tecnológico escolhido para realizar uma intervenção educativa, o conteúdo disponível na tecnologia deve atender às demandas pessoais e ao ritmo de aprendizagem de cada um. ${ }^{(31)}$ Assim, cabe ao profissional decidir qual a tecnologia mais adequada e mais viável a ser utilizada em suas atividades educativas para promoção da saúde de acordo com o seu público.

A utilização de tecnologias educativas associadas à Entrevista Motivacional fortalece a motivação do paciente e seu comprometimento com a mudança de comportamento. ${ }^{(32)}$ Portanto, tecnologias educativas duras, se forem aliadas a tecnologias leves, com o diálogo e a educação em saúde, fortalecerão o vínculo, a saúde dos pacientes, o processo educativo, um atendimento de qualidade e humanizado, bem como irão acarretar em novas possibilidades de interação entre o enfermeiro, o paciente e sua família. ${ }^{(33)}$ Ressalta-se que a Entrevista motivacional parece ser mais eficaz quando é adaptada para coincidir com o nível de autoeficácia e prontidão para mudança de comportamento do indivíduo, como evidenciado no ensaio clínico que avaliou o efeito de uma in- tervenção telefônica na autoeficácia de puérperas na duração e exclusividade da amamentação. ${ }^{(34)}$

Mesmo diante da contribuição dessas tecnologias educacionais, o enfermeiro deve ser o mediador e facilitador nas práticas de educação em saúde, ${ }^{(12)}$ pois sua presença contribui para uma orientação mais clara, objetiva, de fácil entendimento e voltada para realidade, corroborando para a apropriação e o empoderamento das mães. Em consonância, uma pesquisa de validação de uma tecnologia educativa dura, a autora sugeriu associá-la à orientação de profissionais de saúde, visto que a mesma deve ser utilizada de forma complementar, como ferramenta de apoio para reforçar as orientaçôes e jamais deverá superar a função do enfermeiro na sua relação dialógica com seus pacientes. ${ }^{(35)}$

Em face disso, acredita-se que a cartilha e o vídeo educativo associados à EMB influenciam as mães a adotarem atitudes saudáveis, pois estas ao se sentirem mais confiantes, apresentam comportamentos adequados para prevenção de doenças e promoção da saúde dos seus filhos. Ademais, diante de tais consideraçóes, pode-se inferir que intervençóes combinadas tem se mostrado efetivas, pois tanto o uso da cartilha "Você é capaz de prevenir a diarreia no seu filho!" quanto o vídeo "Diarreia Infantil: você é capaz de prevenir” combinado à Entrevista Motivacional Breve foi considerada uma intervenção educativa eficaz para elevar a autoeficácia materna na prevenção da diarreia infantil.

A limitaçáo apresentada por este estudo está relacionada à pesquisa ser unicêntrica e na dificuldade em restabelecer o contato com as mães por telefone com o passar do tempo, reduzindo o tamanho da amostra.

\section{Conclusão}

Evidenciou-se que tanto a intervenção educativa com vídeo e EMB quanto a da cartilha e EMB podem ser utilizadas de maneira eficaz na promoção da autoeficácia materna na prevenção da diarreia infantil. Comprovou-se que o uso dessas duas tecnologias, pautadas no conceito da autoeficácia, foi capaz de elevar os escores da autoeficácia para prevenir a diarreia infantil e reduzir os episódios diar- 
reicos, logo, podem ser utilizadas pelos enfermeiros, como uma ferramenta educativa, a fim de servir de subsídios para orientaçóes às mães. Reforça-se, portanto, que intervençóes que utilizam a EMB podem influenciar no comportamento dos pais em relação à prevenção da diarreia em seus filhos. Neste sentido, acredita-se que as tecnologias educativas combinadas devem ser instituídas nas práticas dos profissionais de saúde, em especial do enfermeiro, auxiliando-os nas estratégias de educação em saúde, com o intuito de fortalecer e elevar a confiança materna na promoção da saúde de seus filhos, bem como a diminuição dos episódios diarreicos.

\section{Agradecimentos}

À Fundação Cearense de Apoio ao Desenvolvimento Científico e Tecnológico - Funcap, ao Conselho Nacional de Desenvolvimento Científico e Tecnológico - CNPq. Às agências financiadoras desta pesquisa e às mães que participaram do estudo.

\section{Colaborações}

Mendes ERR, Sabino LMM, Almeida PC, Melo ESJ, Penha JC, Rocha SS e Barbosa LP declaram que contribuíram com a concepção do estudo, análise e interpretação dos dados, redação do artigo, revisão crítica relevante do conteúdo intelectual e aprovação da versão final a ser publicada.

\section{Referências}

1. United Nations Children's Fund (UNICEF). Estimates Developed by the UN Inter-agency Group for Child Mortality Estimation. United Nations: UNICEF; 2017. [cited Jun 27, 2018]. Available from: http:// www.crianca.mppr.mp.br/arquivos/File/publi/unicef_relatorios/child_ mortality_report_unicef_2017.pdf

2. United Nations Children's Fund (UNICEF). One is too many: ending child deaths from pneumonia and diarrhoea. United Nations: UNICEF; 2016. [cited Apr 2, 2019). Available from: https://www.unicef.org/ publications/index_93020.html

3. Liu L, Oza S, Hogan D, Perin J, Rudan I, Lawn JE, et al. Global, regional, and national causes of child mortality in 2000-13, with projections to inform post-2015 priorities: an updated systematic analysis. Lancet. 2015;385(9966):430-40.
4. World Health Organization (WHO). Source: Global Health Observatory. Genève: WHO; 2017. [cited Aug 27, 2017]. Available from: http://www. who.int/en/

5. Nwaoha AF, Ohaeri CC, Amaechi EC. Prevalence of diarrhoea, and associated risk factors, in children aged 0-5 years, at two hospitals in Umuahia, Abia, Nigeria. Cuad Investig UNED. 2017;9(1):7-14.

6. Suyitno CJ, Tiraphat S, Anye KS. Prevalence and risk factors for diarrhea among children aged less than two years in Central Kalimantan Province, Indonesia. J Pub Health Dev. 2019;17(1):31-45.

7. United Nations Children's Fund (UNICEF); World Health Organization (WHO). World Bank Group; United Nations, Department of Economic and Social Affairs, Population Division; United Nations Economic Commission for Latin America and the Caribbean, Population Division. Levels and trends in child mortality Report [Internet]. New York: UNICEF; Genève: WHO; USA: WORLD BANK; United Nations Population Division; 2015. [cited 2020 Jun 12]. Available from: https://www.unicef.org/ publications/files/Child_Mortality_Report_2015_Web_9_Sept_15.pdf

8. Santos FS, Santos LH, Saldan PC, Santos FC, Leite AM, Mello DF. [Breastfeeding and acute diarrhea among children enrolled in the family health strategy]. Texto Contexto Enferm. 2016; 25(1):e0220015. Portuguese.

9. Luchs A Timenetsky MCS. Group A rotavirus gastroenteritis: postvaccine era, genotypes and zoonotic transmission. einstein (São Paulo). 2016; 14(2):278-87.

10. Luchs A, Cilli A, Morillo SG, Carmona RC, Timenetsky MC. Rotavirus genotypes circulating in brazil, 2007-2012: implications for the vaccine program. Rev Inst Med Trop São Paulo. 2015;57(4):305-13.

11. Imadal KS, Araújo TS, Muniz PT, Pádua VL. Fatores socioeconômicos, higiênicos e de saneamento na redução de diarreia na Amazônia. Rev Saude Publica. 2016;55(77):1-11.

12. Sabino LM, Ferreira ÁM, Mendes ER, Joventino ES, Gubert FD, Penha $\mathrm{JC}$, et al. Validation of primer for promoting maternal self-efficacy in preventing childhood diarrhea. Rev Bras Enferm. 2018;71 Suppl 3:1412-9

13. Ghasemi AA, Talebian A, Masoudi Alavi N, Mousavi GA. Knowledge of mothers in management of diarrhea in under-five children, in Kashan, Iran. Nurs Midwifery Stud. 2013;2(1):158-62.

14. Merga N, Alemayehu T. Knowledge, perception, and management skills of mothers with under-five children about diarrhoeal disease in indigenous and resettlement communities in Assosa District, Western Ethiopia. J Health Popul Nutr. 2015;33(1):20-30.

15. Bandura A. Self-efficacy: toward a unifying theory of behavioral change. Psychol Rev. 1977;84(2):191-215.

16. Bandura A. On the functional properties of perceived self-efficacy revisited. J Manage. 2012;38(1):9-44.

17. Joventino ES, Ximenes LB, da Penha JC, Andrade LC, de Almeida PC. The use of educational video to promote maternal selfefficacy in preventing early childhood diarrhoea. Int J Nurs Pract. 2017;23(3):e12524.

18. Oliveira BS, Oliveira RK, Bezerra JC, Silva MJ, Melo FM, Joventino ES. Crenças e condutas maternas na autoeficácia para prevenir diarreia infantil: estudo transversal. Online Bras J Nurs. 2019;17(1):109-18.

19. Santos FP, Acioli S, Rodrigues VP, Machado JC, Souza MS, Couto TA. Nurse care practices in the Family Health Strategy. Rev Bras Enferm. 2016;69(6):1124-31.

20. Miller WR, Rollnick S. Motivational interviewing: helping people change. 3rd ed. New York (NY): The Guilford Press; 2013. 
21. Hill MM, Hill A. Investigação por questionários. Lisboa: Ed. Sílabos; 2005.

22. Campbell MK, Piaggio G, Elbourne DR, Altman DG. Consort 2010 statement: extension to cluster randomized trials. BMJ. 2012;345:121.

23. Joventino ES, Ximenes LB, Almeida PC, Oriá MO. The maternal selfefficacy scale for preventing early childhood diarrhea: validity and reliability. Public Health Nurs. 2013;30(2):150-8.

24. Oliveira RK, Oliveira BS, Bezerra JC, Silva MJ, Melo FM, Joventino ES. Influence of socio-economic conditions and maternal knowledge in self-effectiveness for prevention of childhood diarrhea. Esc Anna Nery. 2017;21(4):e20160361.

25. Sabino LM, Silva EJ, Vieira AM, Araújo AL, Amaral FG, Penha JC, et al. Educational video for infant diarrhea prevention: randomized clinical trial. Int Arch Med. 2019;9:1-9.

26. Abbasi P, Mohammad-Alizadeh Charandabi S, Mirghafourvand M. Comparing the effect of e-learning and educational booklet on the childbirth self-efficacy: a randomized controlled clinical trial. J Matern Fetal Neonatal Med. 2018;31(5):644-50.

27. De Lepeleere S, De Bourdeaudhuij I, Cardon G, Verloigne M. The effect of an online video intervention 'Movie Models' on specific parenting practices and parental self-efficacy related to children's physical activity, screen-time and healthy diet: a quasi experimental study. BMC Public Health. 2017;17(1):366.
28. Silveira LC, Aliti GB, Da Silva EM, Pimentel RP, Gus M, Rabelo-Silva ER. Effect of motivational interviewing in hypertensive patients (MldNIgHT): study protocol for a randomized controlled trial. Trials. 2019;20(1):414.

29. Dodt RC, Joventino ES, Aquino PS, Almeida PC, Ximenes LB. An experimental study of an educational intervention to promote maternal selfefficacy in breastfeeding. Rev Lat Am Enfermagem. 2015;23(4):725-32.

30. Doak CC, Doak LG, Root JH. Teaching patients with low literacy skills. 2nd ed. Philadelphia: J.B. Lippincott; 1996.

31. Dalmolin A, Girardon-Perlini NM, Coppetti LC, Rossato GC, Gomes JS, Silva ME. Vídeo educativo como recurso para educação em saúde a pessoas com colostomia e familiares. Rev Gaúcha Enferm. 2016; 37(Esp):e68373.

32. Figlie NB, Guimarães LP. The Motivational interview: conversations about changes. Bol Acad Paul Psicol. 2014;34(87):472-89.

33. Pinto TD, Castro DS, Bringuente ME, Sant'Anna HC, Souza TV, Primo CC. Educational animation about home care with premature newborn infants. Rev Bras Enferm. 2018;71 Suppl 4:1604-10.

34. Chaves AF, Ximenes LB, Rodrigues DP, Vasconcelos CT, Monteiro JC, Oriá MO. Telephone intervention in the promotion of self-efficacy, duration and exclusivity of breastfeeding: randomized controlled trial. Rev Lat Am Enfermagem. 2019;27:e3140.

35. Sabino LM, Ferreira AM, Joventino ES, Lima FE, Penha JC, Lima KF, Nascimento LA, Ximenes LB. Elaboration and validation of a reader on childhood diarrhea prevention. Acta Paul Enferm. 2018; 31(3):233-9. 